\title{
Effects of Artificial Sweetener Consumption on Glucose Homeostasis and Its Association with Type 2 Diabetes and Obesity
}

This article was published in the following Dove Press journal: International Journal of General Medicine

\section{Ahmed Abdulrahman Alsunni iD \\ Department of Physiology, College of Medicine, Imam Abdulrahman Bin Faisal University, Dammam, Saudi Arabia}

\begin{abstract}
Artificial sweeteners (ASs) are popular for their characteristic property of providing sweetness with few or no calories. They are frequently consumed to minimize energy intake and to combat obesity and its related adverse health effects. However, since their introduction, concerns have been raised regarding their safety. Extensive research has designed a number of studies to evaluate potential adverse effects, the top among them being interference with glucose homeostasis. Numerous studies have tried to prove that AS may contribute to the development of metabolic diseases including obesity and type 2 diabetes (T2D). The matter remains controversial and a favorite topic of research. The purpose of this review was to identify and discuss the published articles that have examined the effects of AS consumption on glucose homeostasis and its association with T2D and obesity. It was observed that studies have failed to present concrete evidence to establish a link between AS consumption and glucose homeostasis, obesity, or T2D. Most studies have flaws in the study design resulting in haphazard claims with no follow-up studies to confirm reliability. It is concluded that while it is not possible to claim that ASs are metabolically inert, at the moment the haphazard evidence is not enough to link their use with glucose metabolism, obesity or T2D. There is a need to design cohort and case-control studies with reliable sample sizes to establish a cause-effect relationship or to exclude claims of safety problems.
\end{abstract}

Keywords: artificial sweeteners, glucose, diabetes, obesity

\section{Introduction}

Artificial sweeteners (ASs), or non-nutritive sweeteners, are food additives that provide a sweet flavor with zero or low calories; they are used in various products such as foodstuffs, beverages, drugs, and even toothpaste. ${ }^{1}$ They may be derived from plant extracts or manufactured by chemical synthesis. The first AS, benzoic sulfimide, was marketed in the USA in 1879 , by Constantin Fahlberg and was commercialized as saccharin. ${ }^{2}$ Since then, the consumption of ASs has grown substantially, as reported by several studies in adults or children. A 2017 study in the USA showed that AS consumption is $41.4 \%$ among adults and $25.1 \%$ among children. ${ }^{3}$

Currently, six ASs have been approved by the US Food and Drug Administration (FDA): saccharin, acesulfame, aspartame, neotame, sucralose, and advantame. ${ }^{4}$ In 2017, sucralose was the most widely AS consumed; it accounted for one-third of the global market, expected to be worth $\$ 2.8$ billion by $2021 .^{5}$ Figure 1 shows the chemical structures of the approved ASs.
Alsunni

Department of Physiology, College of Medicine, Imam Abdulrahman Bin Faisal University, PO Box 1982, Dammam

3।44I, Saudi Arabia

Tel +966133331188

$\mathrm{Fax}+966133330220$

Email aalsunni@iau.edu.sa
International Journal of General Medicine 2020:13 775-785 
<smiles>O=C1NS(=O)(=O)c2ccccc21</smiles><smiles></smiles>

Saccharin

Acesulfame<smiles>COC(=O)[C@H](Cc1ccccc1)NC(=O)[C@H](N)CC(=O)O</smiles>

Aspartame<smiles>COC(=O)[C@H](Cc1ccccc1)NC(=O)[C@H](CC(=O)O)NCCCc1ccc(OC)c(O)c1</smiles>

Advantame<smiles>OC[C@H]1O[C@@](CO)(O[C@H]2O[C@H](CO)[C@H](O)[C@H]2O)[C@H](O)[C@@H]1O</smiles>

Sucralose<smiles>COC(=O)[C@H](Cc1ccccc1)NC(=O)[C@H](CC(=O)O)NCCC(C)(C)C</smiles>

Neotame

Figure I Chemical structures of the approved artificial sweeteners.

ASs are designed as sugar substitutes to tackle obesity and its subsequent outcomes, including metabolic syndrome, diabetes, and cardiovascular diseases. Yet several concerns have been raised about the safety of these products. In fact, the adverse health effects associated with ASs remain open to discussion among researchers. For instance, the scientific report of the Dietary Guidelines Advisory Committee in 2015 discussed that added sugars should be minimized in the diet and substituted not with ASs, but rather with more healthy choices. ${ }^{6}$ A joint position statement from the American Heart Association and the American Diabetes Association also cautioned against AS intake and stated that there are limited data to conclude whether AS consumption to replace sugar sweeteners benefits body weight, energy balance, or metabolic risk factors. ${ }^{7}$ Meanwhile, Diabetes UK reported that there are adequate data to support that AS consumption improves postprandial blood glucose levels if consumed instead of sugars; moderate consumption of ASs to replace sugars can be a useful strategy to manage body weight. ${ }^{8}$ The purpose of this review is to summarize the available literature investigating the effects of AS consumption on glucose homeostasis and its association with T2D and obesity.

\section{Glucose Homeostasis}

Some clinical trials have observed the effects of ASs on glucose homeostasis. However, the results are contradictory and comparability among these trials is not feasible owing to variations in the sample size, type of AS, whether a placebo was included, exposure time, and outcomes assessed. For example, studies that evaluated the effect of sucralose consumption showed lower, ${ }^{9}$ higher, ${ }^{10}$ or no change in glucose levels. ${ }^{11}$ Likewise, GLP-1 levels were increased following sucralose intake in some studies, 9,12 while other studies reported a reduction ${ }^{13}$ or no change. ${ }^{10}$ Aspartame consumption also showed inconsistent results, with lower ${ }^{14}$ or no change ${ }^{15}$ in plasma glucose levels. 
Other studies investigated the levels of appetite-regulating hormones, including ghrelin, cholecystokinin, and peptide YY, following AS consumption, and showed no significant changes. ${ }^{11,16}$

Overall, as provided in Table 1, the vast majority of clinical trials that have investigated the effects of AS intake on glycemic response observed no significant differences between AS consumption and placebo on various measures of glycemic response, including plasma glucose, insulin, $\mathrm{HbA}_{1 \mathrm{c}}$, and $\mathrm{C}$-peptide. These findings are consistent with the fact that ASs provide few or no calories to the diet. Therefore, based on the available literature, the effect of ASs on glucose homeostasis cannot be established.

\section{Type 2 Diabetes}

ASs are marketed to reduce the risk of metabolic disease, including T2D. Several reports have addressed the relationship between AS consumption and the development of T2D. A dose-response meta-analysis, published in 2020, included 13 cohort studies and investigated the association between artificially sweetened beverages and the risk of T2D; the median follow-up was 8.4 years. The results revealed a linear association between AS intake and T2D risk. The risk increased by $15 \%$ for each $250 \mathrm{~mL} /$ day increase in AS soft drink consumption. However, the study reported considerable heterogeneity and other unmeasurable confounders cannot be excluded. Thus, the results should not be taken for granted. ${ }^{17}$ In another systematic review, four cohorts were included from three observational studies that investigated the association between AS consumption in the form of soft drinks and risk of T2D. The review concluded that there was an increased risk of T2D when $330 \mathrm{~mL}$ of artificially sweetened soft drinks were consumed daily. However, the studies included were observational and significant heterogeneity was reported among the cohorts; therefore, any conclusion should be taken with caution. ${ }^{18}$ In addition, another systematic review analyzed the data from 17 cohorts and found a positive relationship between AS consumption and T2D incidence. However, the authors of this review reported that the evidence of their findings was insufficient and potential bias as well as heterogeneity among cohort studies existed. ${ }^{19}$

Observational studies investigating the relationship between AS consumption and the incidence of T2D showed inconsistent results. Four cohort studies reported a positive association between AS intake and the risk of T2D. Among them, a large cohort study conducted by
Fagherazzi et $\mathrm{al}^{20}$ showed that the risk of T2D was significantly increased among women consuming ASs in the form of packets or tablets for more than 10 years compared to never or rare consumers, when adjusted for body mass index (BMI). However, adiposity cannot be excluded in this study as a confounding factor. Likewise, another study reported a significant positive association between AS intake and the incidence of T2D in women consuming over $600 \mathrm{~mL}$ of artificially sweetened soft drinks for 14 years, with a dose-dependent relationship. However, other independent risk factors for T2D in this study cannot be ruled out. ${ }^{21} \mathrm{~A}$ third study, carried out for 7 years, showed a $67 \%$ greater relative risk for T2D in individuals consuming at least one diet soda per day independent of primary measures of adiposity. However, the possibility of other confounding factors cannot be eliminated from this observational study. ${ }^{22} \mathrm{~A}$ fourth study investigated a limited number of Japanese men for 7 years and reported an increased risk of T2D among subjects who consumed one or more diet sodas daily. The results are not representative of the general population. Also, this study assessed the consumption of AS at the baseline examination and did not consider the possible changes during the 7 years of follow-up. $^{23}$

While some cohort studies and meta-analyses observed a positive relationship between AS consumption and the risk of T2D, other studies reported no association. Bhuphatiraju et $\mathrm{al}^{24}$ investigated the relationship between AS consumption and the incidence of T2D in 39,059 healthy professional men for 20 years. Although a positive association was observed with the development of T2D, in an age-adjusted analysis, the association was no longer significant when adjusted for BMI and total energy intake. These findings indicate that ASs were consumed to reduce weight and health conditions related to obesity, such as diabetes. Similarly, in a case cohort study of 15,384 subjects, there was no significant association between AS consumption and risk of T2D after adjustment for BMI and total energy intake. ${ }^{25}$ A summary of observational cohort studies investigating the association between AS consumption and risk of T2D is provided in Table 2.

A possible explanation for the relationship between AS consumption and risk of T2D that has been observed in some studies might be related to reverse causality. O'Connor et $\mathrm{al}^{26}{ }^{26}$ in a long-term large cohort study of 24,653 adults, concluded that the positive association between AS consumption and T2D may be an artifact of reverse causality. Subjects with higher BMI or a tendency 
Table I Clinical Trials Investigating the Effect of AS Consumption on Glucose Homoeostasis

\begin{tabular}{|c|c|c|c|c|}
\hline $\begin{array}{l}\text { Authors and } \\
\text { Year }\end{array}$ & Study Design & Population and Age & Parameters & Findings \\
\hline $\begin{array}{l}\text { Anton et } \mathrm{al}^{14} \\
2010\end{array}$ & $\begin{array}{l}\text { Single-blind randomized } \\
\text { crossover study }\end{array}$ & $\begin{array}{l}19 \text { participants with normal weight } \\
\text { and } 12 \text { obese subjects } \\
\text { (18-49 years) }\end{array}$ & $\begin{array}{ll}\text { - } & \text { Glucose } \\
\text { - } & \text { Insulin } \\
\text { - Insulinogenic } \\
\text { index }\end{array}$ & $\begin{array}{l}\text { - Reduction in blood glucose and } \\
\text { insulin levels with stevia consump- } \\
\text { tion compared to sucrose } \\
\text { - Lower blood glucose levels with } \\
\text { aspartame consumption at } 20 \text { min } \\
\text { compared to sucrose } \\
\text { - Higher insulinogenic index with } \\
\text { aspartame consumption at I hour }\end{array}$ \\
\hline $\begin{array}{l}\text { Ma et } \mathrm{al}^{41} \\
2010\end{array}$ & $\begin{array}{l}\text { Single-blind randomized } \\
\text { crossover study }\end{array}$ & $\begin{array}{l}10 \text { healthy normal weight subjects } \\
(25-29 \text { years) }\end{array}$ & - Glucose & $\begin{array}{l}\text { Intraduodenal infusion of sucralose } \\
\text { did not change glucose intestinal } \\
\text { absorption or GLP-I secretion } \\
\text { compared to control infusion }\end{array}$ \\
\hline $\begin{array}{l}\text { Brown et al }{ }^{42} \\
2011\end{array}$ & $\begin{array}{l}\text { Double-blind } \\
\text { randomized crossover } \\
\text { study }\end{array}$ & $\begin{array}{l}8 \text { healthy normal weight women } \\
\text { (19-24 years) }\end{array}$ & $\begin{array}{l}\text { - Glucose } \\
\text { - Insulin } \\
\text { - Glucagon } \\
\text { - Ghrelin }\end{array}$ & $\begin{array}{l}\text { No significant changes were described } \\
\text { with sucralose consumption } \\
\text { compared to water }\end{array}$ \\
\hline $\begin{array}{l}\text { Ford et } \mathrm{al}^{43} \\
201 \mathrm{I}\end{array}$ & $\begin{array}{l}\text { Single-blind randomized } \\
\text { crossover study }\end{array}$ & $\begin{array}{l}8 \text { healthy normal weight subjects } \\
(22-27 \text { years })\end{array}$ & $\begin{array}{l}\text { - Glucose } \\
\text { - Insulin } \\
\text { - GLP-I } \\
\text { - PYY }\end{array}$ & $\begin{array}{l}\text { Sucralose consumption did not } \\
\text { change any variables compared to } \\
\text { water }\end{array}$ \\
\hline $\begin{array}{l}\text { Steinert et } \mathrm{al}^{44} \\
20 \mathrm{II}\end{array}$ & $\begin{array}{l}\text { Randomized crossover } \\
\text { study (no blinding) }\end{array}$ & $\begin{array}{l}\text { I2 healthy normal weight subjects } \\
(22-24 \text { years) }\end{array}$ & $\begin{array}{l}\text { - Glucose } \\
\text { - Insulin } \\
\text { - GLP-I } \\
\text { - PYY } \\
\text { - Ghrelin }\end{array}$ & $\begin{array}{l}\text { No significant differences were } \\
\text { observed in any of the parameters } \\
\text { following consumption of aspartame, } \\
\text { acesulfame-k, or sucralose compared } \\
\text { to water }\end{array}$ \\
\hline $\begin{array}{l}\text { Wu et } \mathrm{al}^{45} \\
2012\end{array}$ & $\begin{array}{l}\text { Single-blind randomized } \\
\text { crossover study }\end{array}$ & $\begin{array}{l}10 \text { healthy obese subjects } \\
(25-33 \text { years })\end{array}$ & $\begin{array}{ll}\text { - } & \text { Glucose } \\
\text { - Insulin } \\
\text { - GLP-I } \\
\text { - GIP }\end{array}$ & $\begin{array}{l}\text { Sucralose consumption had no effects } \\
\text { on any parameters }\end{array}$ \\
\hline $\begin{array}{l}\text { Maersk et al }{ }^{16} \\
2012\end{array}$ & $\begin{array}{l}\text { Randomized crossover } \\
\text { study (no blinding) }\end{array}$ & $\begin{array}{l}24 \text { healthy obese subjects } \\
\text { ( } 20-50 \text { years) }\end{array}$ & $\begin{array}{l}\text { - Glucose } \\
\text { - Insulin } \\
\text { - GLP-I } \\
\text { - GIP } \\
\text { - Ghrelin }\end{array}$ & $\begin{array}{l}\text { Aspartame consumption did not } \\
\text { change any variables }\end{array}$ \\
\hline $\begin{array}{l}\text { Brown et al }{ }^{\prime \prime} \\
2012\end{array}$ & $\begin{array}{l}\text { Randomized crossover } \\
\text { study (no blinding) }\end{array}$ & $\begin{array}{l}44 \text { subjects divided into } 3 \text { groups: } 25 \\
\text { healthy controls, } 10 \text { with T2D, and } 9 \\
\text { with type I diabetes } \\
\text { (I2-25 years) }\end{array}$ & $\begin{array}{ll}\text { - } & \text { Glucose } \\
\text { - } & \text { GLP-I } \\
\text { - GIP } \\
\text { - PYY } \\
\text { - C-peptide }\end{array}$ & $\begin{array}{l}\text { - Diet soda consumption before } \\
\text { a glucose load increased GLP-I } \\
\text { secretion in healthy and type I dia- } \\
\text { betic individuals compared to car- } \\
\text { bonated water } \\
\text { - No changes in other parameters }\end{array}$ \\
\hline $\begin{array}{l}\text { Stellingwerff } \\
\text { et } \mathrm{al}^{46} \\
2013\end{array}$ & $\begin{array}{l}\text { Double-blind } \\
\text { randomized crossover } \\
\text { study }\end{array}$ & $\begin{array}{l}23 \text { healthy normal weight men } \\
(22-36 \text { years) }\end{array}$ & - - Glucose & $\begin{array}{l}\text { Sucralose consumption immediately } \\
\text { before exercise had no effects on } \\
\text { glucose and insulin concentrations } \\
\text { during exercise }\end{array}$ \\
\hline
\end{tabular}

(Continued) 
Table I (Continued).

\begin{tabular}{|c|c|c|c|c|}
\hline $\begin{array}{l}\text { Authors and } \\
\text { Year }\end{array}$ & Study Design & Population and Age & Parameters & Findings \\
\hline $\begin{array}{l}\text { Pepino et } \text { al }^{10} \\
2013\end{array}$ & $\begin{array}{l}\text { Randomized crossover } \\
\text { study (no blinding) }\end{array}$ & $\begin{array}{l}17 \text { healthy obese } \\
\text { (34-36 years) }\end{array}$ & $\begin{array}{l}\text { - } \text { Glucose } \\
\text { - Insulin } \\
\text { - Insulin sensitivity } \\
\text { - Insulin clearance } \\
\text { - glucagon } \\
\text { - GLP-I } \\
\text { - GIP } \\
\text { C-peptide }\end{array}$ & $\begin{array}{l}\text { - Sucralose consumption showed } \\
\text { higher glucose, insulin, and } \\
\text { C-peptide concentration, with } \\
\text { a reduction in insulin sensitivity and } \\
\text { insulin clearance } \\
\text { - No differences were found in GIP, } \\
\text { GLP-I, and glucagon }\end{array}$ \\
\hline $\begin{array}{l}\text { Olalde- } \\
\text { Mendoza } \\
\text { et al }{ }^{15} \\
2013\end{array}$ & $\begin{array}{l}\text { Randomized study (no } \\
\text { blinding) }\end{array}$ & $\begin{array}{l}80 \text { obese individuals with T2D } \\
\text { ( } 40-58 \text { years) }\end{array}$ & - Capillary glucose & $\begin{array}{l}\text { Diet soda consumption had no effects } \\
\text { on capillary glucose concentrations }\end{array}$ \\
\hline $\begin{array}{l}\text { Bryant et al } \\
2014\end{array}$ & $\begin{array}{l}\text { Randomized crossover } \\
\text { study (no blinding) }\end{array}$ & $\begin{array}{l}\text { I0 normal weight subjects } \\
\text { (18-24 years) }\end{array}$ & - Glucose & $\begin{array}{l}\text { Consumption of saccharine, } \\
\text { aspartame, or acesulfame-k in } \\
\text { combination with glucose did not } \\
\text { change blood glucose concentrations } \\
\text { compared to glucose alone }\end{array}$ \\
\hline $\begin{array}{l}\text { Temizkan et al9 } \\
2015\end{array}$ & $\begin{array}{l}\text { Single-blind randomized } \\
\text { crossover design }\end{array}$ & $\begin{array}{l}8 \text { newly diagnosed type } 2 \text { diabetic } \\
\text { patients }(51.5 \pm 9.2 \text { years) and } 8 \\
\text { healthy obese subjects }(45.0 \pm 4.1 \\
\text { years) }\end{array}$ & $\begin{array}{ll}\text { - } & \text { Glucose } \\
\text { - Insulin } \\
\text { - } & \text { GLP-I } \\
\text { - } & \text { C-peptide }\end{array}$ & $\begin{array}{l}\text { - Sucralose consumption was asso- } \\
\text { ciated with lower glucose and } \\
\text { higher GLP-I concentrations com- } \\
\text { pared to water consumption in } \\
\text { healthy individuals } \\
\text { - No differences in sucralose or } \\
\text { aspartame on insulin and C-peptide }\end{array}$ \\
\hline $\begin{array}{l}\text { Boyle et al }{ }^{48} \\
2016\end{array}$ & $\begin{array}{l}\text { Single-blind randomized } \\
\text { crossover design }\end{array}$ & $\begin{array}{l}40 \text { healthy normal weight and obese } \\
\text { subjects } \\
\text { ( } 50-65 \text { years) }\end{array}$ & $\begin{array}{l}\text { - } \text { Capillary glucose } \\
\text { - Interstitial } \\
\text { glucose }\end{array}$ & $\begin{array}{l}\text { Sucralose consumption had no effects } \\
\text { on capillary or interstitial glucose } \\
\text { concentrations }\end{array}$ \\
\hline $\begin{array}{l}\text { Dhillon et } \mathrm{al}^{49} \\
2017\end{array}$ & $\begin{array}{l}\text { Single-blind randomized } \\
\text { crossover design }\end{array}$ & $\begin{array}{l}64 \text { obese subjects } \\
\text { (18-50 years) }\end{array}$ & $\begin{array}{l}\text { - Glucose } \\
\text { - Cephalic phase } \\
\text { insulin response }\end{array}$ & $\begin{array}{l}\text { - Significant changes in blood glucose } \\
\text { concentration with sucralose con- } \\
\text { sumption compared to control } \\
\text { - Significant cephalic phase insulin } \\
\text { response following sucralose } \\
\text { consumption }\end{array}$ \\
\hline $\begin{array}{l}\text { Tey et } \mathrm{al}^{50} \\
2018\end{array}$ & $\begin{array}{l}\text { Single-blind randomized } \\
\text { crossover study }\end{array}$ & $\begin{array}{l}32 \text { healthy normal weight men } \\
(2 \mathrm{I}-50 \text { years) }\end{array}$ & $\begin{array}{l}\text { - Postprandial } \\
\text { glucose }\end{array}$ & $\begin{array}{l}\text { - Consumption of low-calorie sweet- } \\
\text { eners was associated with a lower } \\
\text { postprandial glucose concentration } \\
\text { compared to high-calorie sweeteners }\end{array}$ \\
\hline $\begin{array}{l}\text { Crézé et a }\left.\right|^{51} \\
2018\end{array}$ & $\begin{array}{l}\text { Randomized crossover } \\
\text { study (no blinding) }\end{array}$ & $\begin{array}{l}\text { I } 8 \text { healthy normal weight subjects } \\
\text { (age not provided) }\end{array}$ & $\begin{array}{l}\text { - } \text { Glucose } \\
\text { - Insulin } \\
\text { - Ghrelin }\end{array}$ & $\begin{array}{l}\text { - Consumption of artificial swee- } \\
\text { tened beverages was associated } \\
\text { with lower plasma glucose and } \\
\text { insulin concentration compared to } \\
\text { sucrose-sweetened beverages } \\
\text { - Plasma ghrelin showed higher } \\
\text { values in artificially sweetened bev- } \\
\text { erages compared to sucrose- } \\
\text { sweetened beverages }\end{array}$ \\
\hline
\end{tabular}

(Continued) 
Table I (Continued).

\begin{tabular}{|c|c|c|c|c|}
\hline $\begin{array}{l}\text { Authors and } \\
\text { Year }\end{array}$ & Study Design & Population and Age & Parameters & Findings \\
\hline $\begin{array}{l}\text { Farhat et } \mathrm{al}^{52} \\
2019\end{array}$ & $\begin{array}{l}\text { Single-blind randomized } \\
\text { crossover study }\end{array}$ & $\begin{array}{l}30 \text { healthy normal weight and obese } \\
\text { subjects } \\
\text { (16-36 years) }\end{array}$ & $\begin{array}{l}\text { - Postprandial } \\
\text { glucose }\end{array}$ & $\begin{array}{l}\text { - Stevia consumption had no effect } \\
\text { on postprandial glucose }\end{array}$ \\
\hline $\begin{array}{l}\text { Gómez-Arauz } \\
\text { et } \mathrm{al}^{53} \\
2019\end{array}$ & $\begin{array}{l}\text { Randomized placebo- } \\
\text { controlled trial }\end{array}$ & $\begin{array}{l}45 \text { participants divided into } 2 \text { groups: } \\
20 \text { controls ( } 21.55 \pm 2.18 \text { years) and } 25 \\
\text { who ingested sucralose ( } 22 \pm 2.99 \\
\text { years) }\end{array}$ & - Glucose & $\begin{array}{l}\text { - Sucralose consumption showed } \\
\text { higher serum insulin levels at } 30 \text {, } \\
45 \text {, and } 180 \text { min following oral glu- } \\
\text { cose tolerance test. } \\
\text { - No differences were observed for } \\
\text { blood glucose levels }\end{array}$ \\
\hline $\begin{array}{l}\text { Nichol et al }\left.\right|^{54} \\
2020\end{array}$ & $\begin{array}{l}\text { Randomized crossover } \\
\text { study (no blinding) }\end{array}$ & $\begin{array}{l}\text { I } 0 \text { healthy normal weight and II } \\
\text { healthy obese subjects } \\
\text { (23-33 years) }\end{array}$ & $\begin{array}{l}\text { - } \text { Glucose } \\
\text { - Insulin } \\
\text { - } \text { C-peptide } \\
\text { - GIP }\end{array}$ & $\begin{array}{l}\text { - Sucralose consumption elevated } \\
\text { plasma glucose by } 30 \pm 10 \% \text { in lean } \\
\text { and obese subjects compared to } \\
\text { water } \\
\text { - Insulin levels reduced within } 20-40 \\
\text { min of the oral glucose tolerance } \\
\text { test in lean subjects and increased } \\
\text { within } 90-120 \text { min in obese subjects } \\
\text { - C-peptide and GIP did not show } \\
\text { significant differences }\end{array}$ \\
\hline $\begin{array}{l}\text { Bueno-Herná } \\
\text { ndez et } \text { al }^{55} \\
2020\end{array}$ & $\begin{array}{l}\text { Randomized double- } \\
\text { blind placebo- } \\
\text { controlled trial }\end{array}$ & $\begin{array}{l}\text { I } 37 \text { subjects divided into } 3 \text { groups: a) } \\
\text { subjects receiving water as controls, } \\
\text { b) subjects receiving } 48 \mathrm{mg} \text { sucralose, } \\
\text { and c) subjects receiving } 96 \mathrm{mg} \\
\text { sucralose }\end{array}$ & - - insulucose & $\begin{array}{l}\text { Sucralose intake for } 10 \text { weeks } \\
\text { resulted in: } \\
\text { - higher insulin concentrations at } 0 \text {, } \\
30,105 \text { and } 120 \text { min following oral } \\
\text { glucose tolerance test in the group } \\
\text { receiving } 48 \mathrm{mg} \text { sucralose } \\
\text { - higher blood glucose at }-15,0 \text {, and } \\
120 \mathrm{~min} \text { in the group receiving } 48 \\
\text { mg sucralose } \\
\text { - higher area under the curve (AUC) } \\
\text { of insulin in groups receiving } 48 \text { and } \\
96 \mathrm{mg} \text { sucralose }\end{array}$ \\
\hline
\end{tabular}

for weight gain, already at risk of diabetes, consume AS products as a strategy to reduce calorie intake.

In conclusion, the reported studies assessing the risk of T2D reveal divergent results. Reverse causality and residual confounding factors such as adiposity were observed in most of the studies. Therefore, a final conclusion cannot be reached now, and further well-designed human studies, of long duration, evaluating the risk of diabetes are desperately needed.

\section{Obesity}

Various large cohort studies have described a positive dose-dependent relationship between AS consumption and increased BMI. In 2020, Qin et al, ${ }^{17}$ in a meta-analysis including six prospective cohort studies of 26,551 subjects, found that the risk of obesity increased by $21 \%$ for each $250 \mathrm{~mL} /$ day increase in AS soft drink consumption. Likewise, another meta-analysis including three cohort studies of 12,987 participants showed a pooled relative risk of obesity of $1.59 \%$ (95\% CI $1.22-$ 2.08) in subjects consuming artificially sweetened beverages. $^{27}$ Azad et $^{2 l^{28}}$ conducted a meta-analysis of eight cohort studies and concluded that AS consumption was associated with a slightly positive increase in BMI, weight, waist circumference, and incidence of obesity. In a cohort study of 1454 subjects with a median follow-up of 10 years, AS consumers had $0.80 \mathrm{~kg} / \mathrm{m}^{2}$ higher BMI, $2.5 \mathrm{~cm}$ greater waist circumference, and 53\% higher incidence of abdominal obesity compared to non-consumers. ${ }^{29}$ 
Table 2 Observational Cohort Studies Investigating the Association Between AS Consumption and Risk of Development of T2D

\begin{tabular}{|c|c|c|c|}
\hline $\begin{array}{l}\text { Authors and } \\
\text { Year }\end{array}$ & $\begin{array}{l}\text { Number and Age } \\
\text { of Participants }\end{array}$ & $\begin{array}{l}\text { Follow- } \\
\text { up } \\
\text { Period }\end{array}$ & Main Outcomes \\
\hline $\begin{array}{l}\text { Palmer et } \mathrm{al}^{56} \\
2008\end{array}$ & $\begin{array}{l}43,960 \text { women } \\
(21-69 \text { years })\end{array}$ & 4 years & No association was found between diet soft drink consumption and incidence of T2D \\
\hline $\begin{array}{l}\text { Nettleton et al } \\
2009\end{array}$ & $\begin{array}{l}5011 \text { adults } \\
(45-84 \text { years })\end{array}$ & 7 years & Daily diet soda intake was associated with a $67 \%$ higher risk of developing T2D \\
\hline $\begin{array}{l}\text { de Koning et al }{ }^{19} \\
2011\end{array}$ & $\begin{array}{l}40,389 \text { male health } \\
\text { professionals } \\
\text { (40-75 years) }\end{array}$ & 20 years & $\begin{array}{l}\text { Relationship of artificially sweetened beverages consumption and risk of T2D was } \\
\text { observed in the age-adjusted analysis. However, in the multivariate-adjusted analysis no } \\
\text { relationship was found }\end{array}$ \\
\hline $\begin{array}{l}\text { Bhupathiraju } \\
\text { et al }{ }^{24} 2013\end{array}$ & $\begin{array}{l}74,749 \text { female nurses } \\
(30-55 \text { years })\end{array}$ & 24 years & $\begin{array}{l}\text { Significant association was observed between caffeine-free artificially sweetened soft } \\
\text { drinks and incidence of T2D after multivariable adjustment for BMI and energy intake }\end{array}$ \\
\hline $\begin{array}{l}\text { Bhupathiraju } \\
\text { et al }{ }^{24} 2013\end{array}$ & $\begin{array}{l}39,059 \text { healthcare } \\
\text { professional men } \\
(40-75 \text { years })\end{array}$ & 22 years & $\begin{array}{l}\text { No association was found between caffeinated or non-caffeinated artificially sweetened } \\
\text { beverage intake and risk of T2D after multivariable adjustment }\end{array}$ \\
\hline $\begin{array}{l}\text { The InterAct } \\
\text { Consortium } \\
2013\end{array}$ & $\begin{array}{l}34,234 \text { adults } \\
(39-69 \text { years })\end{array}$ & 16 years & $\begin{array}{l}\text { No relationship was observed between artificially sweetened beverage intake and the } \\
\text { incidence of T2D after multivariable adjustment for BMI and energy intake }\end{array}$ \\
\hline $\begin{array}{l}\text { Fagherazzi et } a^{21} \\
2013\end{array}$ & $\begin{array}{l}66,118 \text { women } \\
(46-59 \text { years })\end{array}$ & 14 years & $\begin{array}{l}\text { Significant association was described between high consumption of artificially } \\
\text { sweetened beverages (>630 mL/week) and the development of T2D }\end{array}$ \\
\hline $\begin{array}{l}\text { Sakurai et } \mathrm{al}^{23} \\
2014\end{array}$ & $\begin{array}{l}2037 \text { men } \\
(35-55 \text { years })\end{array}$ & 7 years & $\begin{array}{l}\text { Daily consumption of diet soda was positively associated with increased risk of T2D } \\
\text { after multivariable adjustment }\end{array}$ \\
\hline $\begin{array}{l}\text { O'Connor et } \mathrm{al}^{26} \\
2015\end{array}$ & $\begin{array}{l}24,653 \text { adults } \\
(40-79 \text { years })\end{array}$ & 10.8 years & $\begin{array}{l}\text { Significant association was found between artificially sweetened beverage consumption } \\
\text { and incidence of T2D. Yet, after adjusting for adiposity (BMI and waist circumference) } \\
\text { this became insignificant }\end{array}$ \\
\hline Ma et $\mathrm{al}^{57} 2016$ & $\begin{array}{l}\text { I685 adults } \\
(43-61 \text { years })\end{array}$ & 14 years & No association was found between diet soda intake and increased prediabetes risk \\
\hline $\begin{array}{l}\text { Fagherazzi et al }{ }^{20} \\
2017\end{array}$ & $\begin{array}{l}61,440 \text { women } \\
(46-59 \text { years })\end{array}$ & 18 years & $\begin{array}{l}\text { Significant association was observed between AS consumption in packets or tablets } \\
\text { and development of T2D after adjustment for BMI }\end{array}$ \\
\hline Huang et al ${ }^{58} 2017$ & $\begin{array}{l}64,850 \text { women } \\
(50-79 \text { years })\end{array}$ & 8.4 years & $\begin{array}{l}\text { Consumption of artificially sweetened beverages was associated with a } 21 \% \text { higher risk } \\
\text { of developing T2D }\end{array}$ \\
\hline $\begin{array}{l}\text { Gardener et al }{ }^{59} \\
2018\end{array}$ & $\begin{array}{l}2019 \text { adults } \\
(59-79 \text { years })\end{array}$ & II years & $\begin{array}{l}\text { Strong positive association was found between diet soda intake and the development } \\
\text { of T2D. Yet, after adjusting for BMI this became null }\end{array}$ \\
\hline Jensen et $\mathrm{al}^{60} 2020$ & $\begin{array}{l}1359 \text { adults } \\
(25-60 \text { years })\end{array}$ & 8 years & No association was found between AS consumption and the risk of T2D \\
\hline
\end{tabular}

In children, most of the observational studies demonstrated that AS intake is associated with increased weight. ${ }^{30}$ Moreover, Azad et $\mathrm{al}^{31}$ reported that the daily maternal consumption of AS during pregnancy was associated with a higher infant BMI and a two-fold greater risk of the infant being overweight at the age of 1 year.

Several proposed mechanisms in vitro and in vivo may describe the link between AS intake and obesity. One potential explanation derives from a hypothesis that the sensation of sweetness without calorie absorption may disturb brain metabolic signaling and appetite regulation. ${ }^{32}$ Absence of satiety can encourage more eating. Another mechanism is that AS consumption may activate intestinal and pancreatic sweet taste receptors stimulating GLP-1 and insulin release, as shown in in vitro studies. ${ }^{33}$ Alterations in the gut microbiota have 
also been described as a potential mechanism. Studies of rodent models showed that saccharin intake promotes weight gain and glucose intolerance by changing the physiology of the intestinal microbiota. ${ }^{34,35}$ Another important mechanism that should not be ignored is related to limitations in the study design. Residual confounding and reverse causality in these observational studies may elucidate the relationship between AS intake and obesity. Overweight or obese individuals tend to consume ASs to manage their weight. Likewise, obese people presenting with prediabetes or T2D start consuming ASs to improve their blood glucose control, which causes a false association between AS consumption and the increased risk of developing T2D. This mechanism is supported by the results of several interventional studies that showed no or even a beneficial effect of AS on body weight.

In a meta-analysis of 15 randomized controlled trials (RCTs), the results revealed significant benefits of AS consumption on body weight, BMI, fat mass, and waist circumference compared with sugar intake. The authors concluded that AS consumption as a replacement option for caloric sweeteners may help individuals cope with a weight management plan. ${ }^{36}$ Another meta-analysis of human RCTs of 4 weeks' to 40 months' duration found that AS consumption was associated with reduced body weight compared with a sugar sweetener or water consumption. ${ }^{37}$ Toews et $\mathrm{al}^{38}$ conducted a meta-analysis of RCTs with a study duration of 1 week or longer. The authors found no significant variations in body weight between adults consuming AS compared with those consuming caloric sweeteners or placebo. Subgroup analysis, by body weight level, reported that AS intake by obese or overweight subjects resulted in a reduction of $1.99 \mathrm{~kg}$. However, no change was observed in adults of normal weight. In addition, BMI was 0.6 units lower in individuals consuming AS compared with sucrose consumers. In the meta-analysis mentioned earlier in this section by Azad et $\mathrm{al}^{28}$ the included RCTs did not show a significant correlation between AS consumption and BMI or body weight. The authors concluded that the results from RCTs do not support the proposed benefits of AS for weight control.

Therefore, unlike most prospective observational studies, the meta-analyses of RCT studies suggest that AS consumption has neutral or positive outcomes for weight management. However, methodological limitations in the included studies were apparent and have raised many questions about the outcomes and efficacy of ASs in weight management. Thus, no definite conclusions can be drawn about the effect on body weight of replacing natural sweeteners with ASs.

\section{Future Studies}

While the subject of ASs has generated extensive research, much about ASs has yet to be fully explored. The links between ASs and metabolic diseases remain unclear. At this stage, we are unable to ascertain whether AS consumption has beneficial or adverse effects on health outcomes. Most studies have flaws in study design, study population, sample size, control groups, managing the confounders, use of improper placebo, and interpretation of data. For example, experimental designs using sham feeding, acute exposure, and water as a placebo apparently give inconclusive results. Likewise, gas bubbles in carbonated drinks may act as a confounder in some study designs, resulting in a lack of consistent results among different groups. Thus, it is of paramount importance to design long-term cohort studies along the pattern of the Framingham Study to determine cause-and-effect relationships between AS consumption and the prevalence of different metabolic diseases. Likewise, case-control studies could be designed to establish cause-and-effect relationships in obese individuals presenting with T2D. In addition, future studies should attempt to explain the effects of different AS independently since ASs may differ in their physiological impacts. One study found that different ASs (saccharine, sucralose, and aspartame) had different effects on body weight over 3 months. ${ }^{39}$ Thus, the type and dose of AS used should be documented clearly in all studies.

Besides investigating the effects of AS intake on healthy individuals, research should also be directed toward diseased populations and other subgroups, such as pregnant women and their infants. Exposure of babies to ASs via breast milk has been postulated to have potential adverse outcomes in adult life. ${ }^{40}$ Further studies are needed to explore this matter in detail.

\section{Conclusion}

AS consumption is associated with obesity and T2D in observational cohort studies, but the findings are questionable since reverse causality and residual confounders cannot be excluded. A majority of interventional clinical trials observe neutral or even beneficial effects of AS when consumed in the context of a weight-loss program. Further well-designed studies that mimic real-life 
consumption are needed to explore the long-term specific effects of the different ASs available.

Based on the available data, it is not possible to claim that ASs are metabolically inert; however, current evidence is not sufficient to link their use with glucose metabolism, obesity, or T2D.

\section{Disclosure}

The author reports no conflicts of interest in this work.

\section{References}

1. Gardener H, Elkind MS. Artificial sweeteners, real risks. Am Heart Assoc. 2019.

2. Remsen I. On the oxidation of substitution products of aromatic hydrocarbons. J Am Chem Soc. 1879;1(4):115-116. doi:10.1021/ ja02144a604

3. Sylvetsky AC, Jin Y, Clark EJ, Welsh JA, Rother KI, Talegawkar SA. Consumption of low-calorie sweeteners among children and adults in the United States. J Acad Nutr Diet. 2017;117(3):441-8. e2. doi:10.1016/j.jand.2016.11.004

4. FDA. High-intensity sweeteners; 2014. Available from: https://www. fda.gov/food/food-additives-petitions/high-intensity-sweeteners. Accessed August 17, 2020.

5. Busness Wire. Global food market-growth, trends, forecast for the period (2015-2020); 2017. Available from: https://www.businesswire. com/news/home/20170331005203/en/Global-Zero-Calorie-SweetenerMarket-Projected-Worth-USD. Accessed August 17, 2020.

6. Millen BE, Abrams S, Adams-Campbell L, et al. The 2015 dietary guidelines advisory committee scientific report: development and major conclusions. Adv Nutr. 2016;7(3):438-444. doi:10.3945/an.116.012120

7. Gardner C, Wylie-Rosett J, Gidding SS, et al. Nonnutritive sweeteners: current use and health perspectives: a scientific statement from the American Heart Association and the American diabetes Association. Circulation. 2012;126(4):509-519. doi:10.1161/CIR.0b0 $13 \mathrm{e} 31825 \mathrm{c} 42 \mathrm{ee}$

8. Dyson P, Twenefour D, Breen C, et al. Diabetes UK evidence-based nutrition guidelines for the prevention and management of diabetes. Diabet Med. 2018;35(5):541-547. doi:10.1111/dme.13603

9. Temizkan S, Deyneli O, Yasar M, et al. Sucralose enhances GLP-1 release and lowers blood glucose in the presence of carbohydrate in healthy subjects but not in patients with type 2 diabetes. Eur J Clin Nutr. 2015;69(2):162-166. doi:10.1038/ejcn.2014.208

10. Pepino MY, Tiemann CD, Patterson BW, Wice BM, Klein S. Sucralose affects glycemic and hormonal responses to an oral glucose load. Diabetes Care. 2013;36(9):2530-2535. doi:10.2337/dc12-2221

11. Brown RJ, Walter M, Rother KI. Effects of diet soda on gut hormones in youths with diabetes. Diabetes Care. 2012;35(5):959-964. doi:10.2337/dc11-2424

12. Ohtsu $Y$, Nakagawa $Y$, Nagasawa $M$, Takeda S, Arakawa $H$, Kojima I. Diverse signaling systems activated by the sweet taste receptor in human GLP-1-secreting cells. Mol Cell Endocrinol. 2014;394(1-2):70-79. doi:10.1016/j.mce.2014.07.004

13. Hall W, Millward D, Rogers P, Morgan L. Physiological mechanisms mediating aspartame-induced satiety. Physiol Behav. 2003;78(4 5):557-562. doi:10.1016/S0031-9384(03)00034-9

14. Anton SD, Martin CK, Han H, et al. Effects of stevia, aspartame, and sucrose on food intake, satiety, and postprandial glucose and insulin levels. Appetite. 2010;55(1):37-43. doi:10.1016/j.appet.2010.03.009

15. Olalde-Mendoza L, Moreno-González YE. Modification of fasting blood glucose in adults with diabetes mellitus type 2 after regular soda and diet soda intake in the state of Queretaro, Mexico. Arch Latinoam Nutr. 2013;63(2):142-147.
16. Maersk M, Belza A, Holst JJ, et al. Satiety scores and satiety hormone response after sucrose-sweetened soft drink compared with isocaloric semi-skimmed milk and with non-caloric soft drink: a controlled trial. Eur J Clin Nutr. 2012;66(4):523-529. doi:10.1038/ ejen.2011.223

17. Qin P, Li Q, Zhao Y, et al. Sugar and artificially sweetened beverages and risk of obesity, type 2 diabetes mellitus, hypertension, and allcause mortality: a dose-response meta-analysis of prospective cohort studies. Springer. 2020.

18. Greenwood D, Threapleton D, Evans C, et al. Association between sugarsweetened and artificially sweetened soft drinks and type 2 diabetes: systematic review and dose-response meta-analysis of prospective studies. Br J Nutr. 2014;112(5):725-734. doi:10.1017/S0007114514001329

19. De Koning L, Malik VS, Rimm EB, Willett WC, Hu FB. Sugarsweetened and artificially sweetened beverage consumption and risk of type 2 diabetes in men. Am J Clin Nutr. 2011;93(6):1321-1327. doi:10.3945/ajen.110.007922

20. Fagherazzi G, Gusto G, Affret A, et al. Chronic consumption of artificial sweetener in packets or tablets and type 2 diabetes risk: evidence from the E3N-European prospective investigation into cancer and nutrition study. Ann Nutr Metab. 2017;70(1):51-58. doi: $10.1159 / 000458769$

21. Fagherazzi G, Vilier A, Saes Sartorelli D, Lajous M, Balkau B, Clavel-Chapelon F. Consumption of artificially and sugar-sweetened beverages and incident type 2 diabetes in the etude epidémiologique auprès des femmes de la mutuelle générale de l'education nationaleEuropean prospective investigation into cancer and nutrition cohort. Am J Clin Nutr. 2013;97(3):517-523. doi:10.3945/ajcn.112.050997

22. Nettleton JA, Lutsey PL, Wang Y, Lima JA, Michos ED, Jacobs DR. Diet soda intake and risk of incident metabolic syndrome and type 2 diabetes in the Multi-Ethnic Study of Atherosclerosis (MESA). Diabetes Care. 2009;32(4):688-694. doi:10.2337/dc08-1799

23. Sakurai M, Nakamura K, Miura K, et al. Sugar-sweetened beverage and diet soda consumption and the 7-year risk for type 2 diabetes mellitus in middle-aged Japanese men. Eur $J$ Nutr. 2014;53 (1):251-258. doi:10.1007/s00394-013-0523-9

24. Bhupathiraju SN, Pan A, Malik VS, et al. Caffeinated and caffeine-free beverages and risk of type 2 diabetes. Am J Clin Nutr. 2013;97(1):155-166.

25. InterAct Consortium. Consumption of sweet beverages and type 2 diabetes incidence in European adults: results from EPIC-InterAct. Diabetologia. 2013;56(7):1520-1530. doi:10.1007/s00125-013-2899-8

26. O'Connor L, Imamura F, Lentjes MA, Khaw K-T, Wareham NJ, Forouhi NG. Prospective associations and population impact of sweet beverage intake and type 2 diabetes, and effects of substitutions with alternative beverages. Diabetologia. 2015;58(7):1474-1483. doi:10.1007/s00125-015-3572-1

27. Ruanpeng D, Thongprayoon C, Cheungpasitporn W, Harindhanavudhi T. Sugar and artificially sweetened beverages linked to obesity: a systematic review and meta-analysis. QJM. 2017;110(8):513-520. doi:10.1093/qjmed/hcx068

28. Azad MB, Abou-Setta AM, Chauhan BF, et al. Nonnutritive sweeteners and cardiometabolic health: a systematic review and meta-analysis of randomized controlled trials and prospective cohort studies. CMAJ. 2017;189(28):E929-E39. doi:10.1503/cmaj.161390

29. Chia CW, Shardell M, Tanaka T, et al. Chronic low-calorie sweetener use and risk of abdominal obesity among older adults: a cohort study. PLoS One. 2016;11(11):e0167241. doi:10.1371/journal.pone.016 7241

30. Durán Agüero S, Angarita Davila L, Escobar Contreras M, Rojas Gomez D, de Assis Costa J. Noncaloric sweeteners in children: A controversial theme. Biomed Res Int. 2018;2018.

31. Azad MB, Sharma AK, de Souza RJ, et al. Association between artificially sweetened beverage consumption during pregnancy and infant body mass index. JAMA Pediatr. 2016;170(7):662-670. doi:10.1001/jamapediatrics.2016.0301 
32. Sylvetsky AC, Rother KI. Nonnutritive sweeteners in weight management and chronic disease: a review. Obesity. 2018;26(4):635-640. doi:10.1002/oby.22139

33. Jang H-J, Kokrashvili Z, Theodorakis MJ, et al. Gut-expressed gustducin and taste receptors regulate secretion of glucagon-like peptide-1. Proc Natl Acad Sci. 2007;104(38):15069-15074. doi:10.1073/pnas.0706890104

34. Suez J, Korem T, Zeevi D, et al. Artificial sweeteners induce glucose intolerance by altering the gut microbiota. Nature. 2014;514 (7521):181-186. doi:10.1038/nature13793

35. Suez J, Korem T, Zilberman-Schapira G, Segal E, Elinav E. Noncaloric artificial sweeteners and the microbiome: findings and challenges. Gut Microbes. 2015;6(2):149-155. doi:10.1080/ 19490976.2015.1017700

36. Miller PE, Perez V. Low-calorie sweeteners and body weight and composition: a meta-analysis of randomized controlled trials and prospective cohort studies. Am J Clin Nutr. 2014;100(3):765-777. doi:10.3945/ajen.113.082826

37. Rogers PJ, Hogenkamp PS, De Graaf C, et al. Does low-energy sweetener consumption affect energy intake and body weight? A systematic review, including meta-analyses, of the evidence from human and animal studies. Int $J$ Obes. 2016;40(3):381-394. doi:10.1038/ijo.2015.177

38. Toews I, Lohner S, de Gaudry DK, Sommer H, Meerpohl JJ. Association between intake of non-sugar sweeteners and health outcomes: systematic review and meta-analyses of randomised and non-randomised controlled trials and observational studies. BMJ. 2019;364.

39. Higgins KA, Considine RV, Mattes RD. Aspartame consumption for 12 weeks does not affect glycemia, appetite, or body weight of healthy, lean adults in a randomized controlled trial. $J$ Nutr. 2018;148(8):650-657.

40. Sylvetsky AC, Gardner AL, Bauman V, et al. Nonnutritive sweeteners in breast milk. $J$ Toxicol Environ Health. 2015;78 (16):1029-1032. doi:10.1080/15287394.2015.1053646

41. Ma J, Chang J, Checklin HL, et al. Effect of the artificial sweetener, sucralose, on small intestinal glucose absorption in healthy human subjects. Br J Nutr. 2010;104(6):803-806. doi:10.1017/S0007 114510001327

42. Brown AW, Brown MMB, Onken KL, Beitz DC. Short-term consumption of sucralose, a nonnutritive sweetener, is similar to water with regard to select markers of hunger signaling and short-term glucose homeostasis in women. Nutr Res. 2011;31(12):882-888.

43. Ford H, Peters V, Martin N, et al. Effects of oral ingestion of sucralose on gut hormone response and appetite in healthy normal-weight subjects. Eur J Clin Nutr. 2011;65(4):508-513. doi:10.1038/ejen.2010.291

44. Steinert RE, Frey F, Töpfer A, Drewe J, Beglinger C. Effects of carbohydrate sugars and artificial sweeteners on appetite and the secretion of gastrointestinal satiety peptides. Br $J$ Nutr. 2011;105 (9):1320-1328.

45. Wu T, Zhao BR, Bound MJ, et al. Effects of different sweet preloads on incretin hormone secretion, gastric emptying, and postprandial glycemia in healthy humans. Am J Clin Nutr. 2012;95(1):78-83. doi:10.3945/ajen.111.021543

46. Stellingwerff T, Godin J-P, Beaumont M, et al. Effects of pre-exercise sucralose ingestion on carbohydrate oxidation during exercise. Int J Sport Nutr Exerc Metab. 2013;23(6):584-592.
47. Bryant CE, Wasse LK, Astbury N, Nandra G, McLaughlin JT. Nonnutritive sweeteners: no class effect on the glycaemic or appetite responses to ingested glucose. Eur J Clin Nutr. 2014;68 (5):629-631. doi:10.1038/ejen.2014.19

48. Boyle NB, Lawton CL, Allen R, Croden F, Smith K, Dye L. No effects of ingesting or rinsing sucrose on depleted self-control performance. Physiol Behav. 2016;154:151-160. doi:10.1016/j. physbeh.2015.11.019

49. Dhillon J, Lee JY, Mattes RD. The cephalic phase insulin response to nutritive and low-calorie sweeteners in solid and beverage form. Physiol Behav. 2017;181:100-109. doi:10.1016/j.physbeh.20 17.09.009

50. Tey SL, Salleh N, Henry CJ, Forde CG. Effects of consuming preloads with different energy density and taste quality on energy intake and postprandial blood glucose. Nutrients. 2018;10(2):161. doi:10.3390/nu10020161

51. Crézé C, Candal L, Cros J, et al. The impact of caloric and non-caloric sweeteners on food intake and brain responses to food: a randomized crossover controlled trial in healthy humans. Nutrients. 2018;10(5):615. doi:10.3390/nu10050615

52. Farhat G, Berset V, Moore L. Effects of stevia extract on postprandial glucose response, satiety and energy intake: a three-arm crossover trial. Nutrients. 2019;11(12):3036. doi:10.3390/nu11123036

53. Gómez-Arauz AY, Bueno-Hernández N, Palomera LF, et al. A single $48 \mathrm{mg}$ sucralose sip unbalances monocyte subpopulations and stimulates insulin secretion in healthy young adults. J Immunol Res. 2019;2019.

54. Nichol AD, Salame C, Rother KI, Pepino MY. Effects of sucralose ingestion versus sucralose taste on metabolic responses to an oral glucose tolerance test in participants with normal weight and obesity: a randomized crossover trial. Nutrients. 2020;12(1):29. doi:10.3390/ nu12010029

55. Bueno-Hernández N, Esquivel-Velázquez $\mathrm{M}$, Alcántara-Suárez R, et al. Chronic sucralose consumption induces elevation of serum insulin in young healthy adults: a randomized, double blind, controlled trial. Nutr J. 2020;19:1-12.

56. Palmer JR, Boggs DA, Krishnan S, Hu FB, Singer M, Rosenberg L. Sugar-sweetened beverages and incidence of type 2 diabetes mellitus in African American women. Arch Intern Med. 2008;168 (14):1487-1492. doi:10.1001/archinte.168.14.1487

57. Ma J, Jacques PF, Meigs JB, et al. Sugar-sweetened beverage but not diet soda consumption is positively associated with progression of insulin resistance and prediabetes. J Nutr. 2016;146(12):2544-2550. doi: $10.3945 /$ jn. 116.234047

58. Huang M, Quddus A, Stinson L, et al. Artificially sweetened beverages, sugar-sweetened beverages, plain water, and incident diabetes mellitus in postmenopausal women: the prospective women's health initiative observational study. Am J Clin Nutr. 2017;106(2):614-622. doi:10.3945/ajen.116.145391

59. Gardener H, Moon YP, Rundek T, Elkind MS, Sacco RL. Diet Soda and sugar-sweetened soda consumption in relation to incident diabetes in the Northern Manhattan study. Curr Dev Nutr. 2018;2(5): nzy008. doi:10.1093/cdn/nzy008

60. Jensen PN, Howard BV, Best LG, et al. Associations of diet soda and non-caloric artificial sweetener use with markers of glucose and insulin homeostasis and incident diabetes: the strong heart family study. Eur J Clin Nutr. 2020;74(2):322-327. doi:10.1038/s41430019-0461-6 


\section{Publish your work in this journal}

The International Journal of General Medicine is an international, peer-reviewed open-access journal that focuses on general and internal medicine, pathogenesis, epidemiology, diagnosis, monitoring and treatment protocols. The journal is characterized by the rapid reporting of reviews, original research and clinical studies across all disease areas. The manuscript management system is completely online and includes a very quick and fair peer-review system, which is all easy to use. Visit http://www.dovepress.com/ testimonials.php to read real quotes from published authors.

Submit your manuscript here: https://www.dovepress.com/international-journal-of-general-medicine-journal 